\title{
CFD-Based Investigation of the Effectiveness of a Novel Longitudinal Ventilation Concept in Tunnels
}

\author{
Merve Altay, Ali Surmen \\ Mechanical Engineering, Bursa Technical University \\ Turkey
}

\section{Extended Abstract}

The construction and complexity of road tunnels are substantially changing throughout the world to meet the need of changing traffic conditions. As a result of catastrophic multiple-death fires occurred in the past years in highway tunnels, the issue of fire safety in road tunnels has gained high importance and visibility throughout the world. However, it is really hard to conduct experimental or numerical studies to ensure fire safety in tunnels because of such reasons as being under the effect of so many dynamic parameters, large tunnel sizes, the heat feedback from the surrounding environment and the effect of natural ventilation on fire, etc. Despite all these restrictions, there are effective fire safety solutions providing high-level protection for people and reducing the risk of structural damage. However, that proposed solutions, most of which need extra space apart from the operational tunnel area, are too different systems from the current and aging tunnels in terms of construction. On the other hand, conventional longitudinal ventilation systems (CLVS) are still being commonly used, in spite of being insufficient to keep the fire, with high heat release rate (HRR), under control.

In this study, a new longitudinal ventilation design concept which could be integrated to the current CLVS was developed based on an idea whether it would be more effective and economical to make some improvements in current systems instead of designing a totally new concept. Within the scope of this, a horse shoe shape cross-sectioned tunnel with CLVS was modelled using the physical conditions of the tunnel of a reference study and the efficiencies of the existing ventilation system and the new design concept for different cases compared by using FDS (Fire Dynamics Simulator) simulation results. In this new design concept fire was detected by sensors and then automated longitudinal ventilation system with jet fans was activated in optimum ventilation condition. This could be assumed to be a case study conducted to test this new concept performance changing the operation orders and charge velocities and positions of jet fans. Numerical analysis results demonstrated that the developed system meets the most recent tunnel safety standards. Even if such severe fires as tanker fires occur in tunnels, limit values of toxic concentration, air velocity and air temperature that human body can tolerate, tunnel facilities and ventilation system can endure didn't exceed. Briefly, satisfactory results in terms of tunnel fire safety were observed from the CFD simulations. Although investment cost of this new system seems more expensive than that of conventional ventilation or smoke extraction systems at first sight, when it is considered sufficient systems to keep that much severe fire under control, the investment cost of it is affordable.

Keywords: Longitudinal Ventilation Systems, Tunnel Fires, Smoke Extraction Systems 